\title{
Exclusive studies on short range correlations in nuclei
}

\author{
Maria Patsyuk ${ }^{1, *}$, Or Hen ${ }^{1,}$, and Eliezer Piasetzky ${ }^{2}$, \\ ${ }^{1}$ Massachusetts Institute for Technology, Cambridge, MA, USA \\ ${ }^{2}$ Tel-Aviv University, Tel-Aviv, Israel
}

\begin{abstract}
Short Range Correlations (SRC) are brief fluctuations of high relative momentum nucleon pairs. Properties of SRC have important consequences for nuclear physics, high energy physics, atomic physics, and astrophysics. SRC pairs form some of the densest states of cold matter achievable on Earth, making them an ideal system to study the partonic and nucleonic degrees of freedom in nuclear systems. Hard exclusive breakup reactions, where high-energy probes scatter on SRC pairs, are used to study such properties of SRC pairs as isospin decomposition, nuclear mass and asymmetry dependence, c.m. momentum distribution. Thomas Jefferson National Accelerator Facility (JLab) plays a key role in the SRC program. CLAS (CEBAF Large Acceptance Spectrometer), located in Hall B at JLab, has almost $4 \pi$ coverage and is capable of measuring exclusive reactions of the type $\mathrm{A}(\mathrm{e}, \mathrm{e}$ 'pp). We will discuss the recent experimental results from JLab and future experiments planned at JLab as well as at JINR.
\end{abstract}

\section{Basics of short range correlations}

Short Range Correlations are brief fluctuations of two nucleons with high and opposite momenta, where each of them is higher than the Fermi momentum for the given nucleus, and the center of mass momentum is lower than the Fermi momentum. The fraction of high momentum nucleon in nuclei is about $20-25 \%$. It was shown experimentally, that almost all high momentum nucleons belong to SRC pairs. The presence of SRC pairs within any nucleus and their properties are important for understanding the nucleon-nucleon interaction and the interplay between partonic and nucleonic degrees of freedom in nuclear systems.

\section{$2 \mathrm{SRC}$ in exclusive hard scattering reactions}

The study of SRCs using exclusive reactions has a long history, but here we focus only on exclusive measurements performed with high energy probes and large momentum transfer (hard reactions). The kinematics of the reaction of interest assuming a fixed nuclear target is the following: the beam probe interacts with a single nucleon in the target nucleus, so that both the scattered particle and the struck nucleon leave the nucleus and get detected. In case an SRC nucleon was hit, the correlated partner emerges as well. This type of experiments were done at BNL (a proton beam on a carbon target), followed by a series of experiments at JLab (an electron beam on nuclear targets). This year we started a second generation of hard

\footnotetext{
*e-mail: mpatsyuk@mit.edu
} 
exclusive SRC experiments in Dubna, where inverse kinematics is used: an ion beam hits a liquid hydrogen target. In this case the residual nuclear system is accessible for identification for the first time.

Exclusive hard scattering reactions are a perfect tool to study SRC properties. Modern detector systems with large angular coverage and ability to register both charged and neutral particles, such as CLAS 6 in Fig. 1, allow detection of all particles in the final state. The open electron trigger of CLAS 6 allowed search for particular signatures in the stored data.

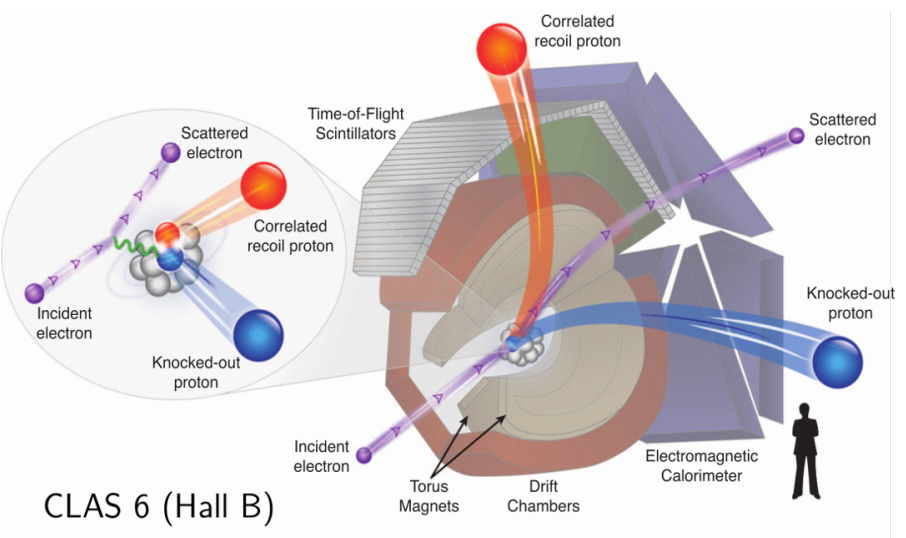

Figure 1. The CLAS spectrometer. The reaction of hard knockout of two nucleons from the target nucleus by an electron probe is shown. All three final state particles are detected by the spectrometer, which ensures the exclusiveness of the measurement

The resolving power of the measurement is determined by its momentum transfer, and the interpretation relies on the theoretical modeling of the interaction that should account for all possible mechanisms that lead to the same measured final state. In addition to quasielastic (QE) scattering from a single nucleon, the full reaction mechanism could include contributions from Meson-Exchange Current, Isobar Currents, and elastic and inelastic nucleon rescattering (final state interactions, FSI). In the case of high initial momentum of the struck nucleon, elastic FSI include re-scattering between the nucleons of the pair and/or with the residual system. The relative contribution of these reaction mechanisms, as compared to the QE reaction of interest, strongly depends on the reaction kinematics. Minimizing non-QE reaction mechanisms also reduces their interference with the $\mathrm{QE}$ reaction. The events, where the knocked-out nucleon predominantly belonged to an SRC pair can be selected by requiring a large Bjorken scaling parameter $x_{B}=Q^{2} / 2 m_{p} \omega \geq 1.2$ (where $Q^{2}=\vec{q}^{2}-\omega^{2}, \vec{q}$ and $\omega$ are the three-momentum and energy, respectively, transferred to the nucleus, and $m_{p}$ is the proton mass) and large momentum transfer $Q^{2}>1.8 \mathrm{GeV}^{2}$. The events of interest have limited reconstructed missing mass of the two-nucleon system $m_{\text {miss }}=\left[\left(\omega+2 m_{p}+E_{p}\right)^{2}-p_{\text {miss }}^{2}\right]^{1 / 2} \leq 1.1 \mathrm{GeV}$, here $E_{p}$ is the total energy of the leading proton, $\vec{p}_{\text {miss }}=\vec{p}_{p}-\vec{q}$, where $\vec{p}_{p}$ is the measured proton momentum. The events where the leading proton absorbed transferred momentum can be identified by requiring that its momentum $\vec{p}_{p}$ is within $25^{\circ}$ of $\vec{q}$.

\section{Highlights from the modern SRC picture}

The first exclusive two-nucleon knockout experiments, measuring the ${ }^{12} C(p, 2 p n)$ and ${ }^{12} C\left(e, e^{\prime} p N\right)$ reactions were done at BNL and JLab, respectively [1-4]. These experiments 
scattered 5-9 GeV/c protons (BNL) and electrons (JLab) off high initial momentum (300 $\leq p_{\text {miss }} \leq 600 \mathrm{MeV} / \mathrm{c}$ ) protons in ${ }^{12} \mathrm{C}$ and looked for a correlated recoil nucleon. The JLab experiment measured both proton and neutron recoils, whereas the BNL experiment measured only recoiling neutrons. The BNL measurement showed that $92 \%$ of high momentum leading protons have a recoil neutron emitted in the opposite direction (see Fig. 2a). The shape of the recoil nucleon opening angle distribution, shown in Fig. 2b, allowed extracting the pair c.m. motion. This motion can be described by a Gaussian distribution in each direction, with $\sigma=143 \pm 17 \mathrm{MeV} / c$ (BNL) and $\sigma=136 \pm 20 \mathrm{MeV} / c$ (JLab). These values are in overall agreement with theoretical calculations $[15,16]$. The electron and proton reactions are characterized by completely different operators and FSI mechanisms, therefore, the agreement of their c.m. momentum distributions validated the consistent treatment of FSI in these measurements.

a)

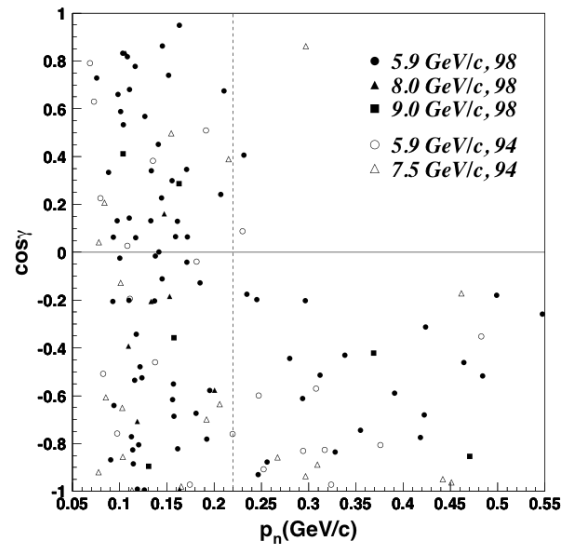

b)

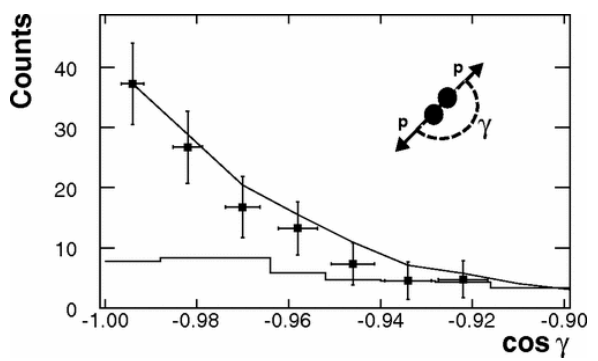

Figure 2. Distribution of the relative angle $(\gamma)$ between the reconstructed initial momentum of the knockout proton and the recoil neutron as a function of the recoil neutron momentum $p_{n}$ (a). The vertical dashed line at $k_{F}=225 \mathrm{MeV} / c$ marks the border between the regions with isotropic $\left(p_{n}<k_{F}\right)$ and correlated $\left(p_{n}>k_{F}\right)$ distributions. (b) projection on $\cos (\gamma)$ axis for scattering off $\sim 500 \mathrm{MeV} / c$ initial momentum protons. Pictures are adapted from $[2,3]$

Fig. 3a shows the extracted ratio of two-nucleon knockout (proton-neutron and protonproton) to single proton knockout events (circles and triangles) and the ratio of proton-proton to proton-neutron two-nucleon knockout events (the red square). The ratios are corrected for finite acceptance of the detectors and are shown as a function of the reconstructed initial momentum of knocked-out protons $p_{\text {miss }}$. The ratio of two-nucleon knockout to single proton knockout is directly related to the fraction of high-momentum protons that are in SRC pairs. Within statistical uncertainties of about $10 \%$ all events with a single knocked out proton with initial momentum of $300 \leq p_{\text {miss }} \leq 600 \mathrm{MeV} / c$ have a recoil nucleon. The ratio between recoil protons and neutrons was found to be approximately $1: 10$, which corresponds to 20 times more $n p$-SRC pairs than $p p$-SRC pairs in ${ }^{12} C$. The observed dominance of neutronproton pairs over proton-proton and neutron-neutron ones was associated with the dominance of the tensor part of the nucleon-nucleon interaction at these initial momenta. Recent CLAS-6 data helped to establish np-dominance for a wide range of nuclei (see Fig. 3b).

The modern state of the SRC studies can be summarized as: 
a)

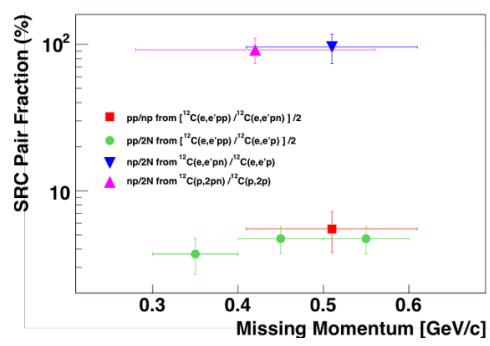

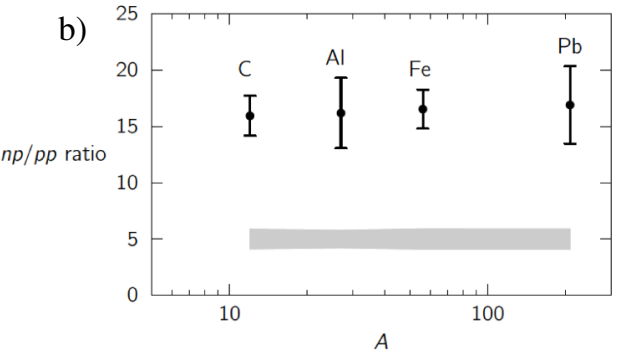

Figure 3. a): The ratio of ${ }^{12} C\left(e, e^{\prime} p N\right)$ events to ${ }^{12} C\left(e, e^{\prime} p\right)$ events as a function of the reconstructed initial momentum of the knockout proton from the ${ }^{12} C\left(e, e^{\prime} p\right)$ reaction. The triangles mark knock out of a proton and a neutron by the proton $(\triangle$, BNL result) and electron $(\nabla)$ probes. The circles mark the knockout of two protons. The square shows the ${ }^{12} C\left(e, e^{\prime} p p\right) /{ }^{12} C\left(e, e^{\prime} p\right)$ ratio. Picture is adapted from [4] b) Recent CLAS-6 data analysis established the np-dominance for large range of $A$

- SRC exist in nuclei and account for about $20 \%$ of nucleons and almost all high momentum $\left(p_{\text {miss }}>k_{F}\right)$ nucleons;

- Nucleons within the pair have high momentum $\left(p_{\text {miss }}>k_{F}\right)$, and the c.m. momentum of the pair is low $\left(p_{c . m .}<k_{F}\right)$;

- $n p$-pairs are about 10 times more likely than $p p$ - or $n n$-pairs ( $n p$-dominance effect);

- Tensor, spin-dependent interaction within SRC pair.

The areas of immediate interest at this point may include a detailed study of $n p$ dominance for asymmetric and neutron-rich nuclei, investigation of the nucleon structure modifications inside the nucleus. Also, the SRC-formation process is of particular interest, as it might provide valuable insights about the nucleon-nucleon repulsive interaction at short distances. The next section describes the recent analysis results, providing some insights to these aspects of SRC.

\section{Recent results from the CLAS-6 data analysis}

The work presented in below is collected using a $5.014 \mathrm{GeV}$ electron beam incident on deuterium, carbon, aluminum, iron, and lead targets. The CLAS spectrometer was used to detect the scattered electron and any associated hadrons knocked out in the interaction (see Fig. 1). CLAS uses a toroidal magnetic field and six independent sets of drift chambers, time-offlight scintillator counters, Cherenkov counters, and electromagnetic calorimeters, covering scattering angles from about $8^{\circ}$ to $140^{\circ}$ for charged particle identification and track reconstruction. The neutrons were identified by observing interactions in the forward electromagnetic calorimeters (covering about $8^{\circ}$ to $45^{\circ}$ ) with no associated charged particle tracks in the drift chambers. The neutron detection efficiency as a function of angle and momentum was measured at the same time using the $d\left(e, e^{\prime} p \pi^{+} \pi^{-} n\right)$ reaction. All events with a scattered electron detected in both the electromagnetic calorimeter and the Cherenkov counter, along with any other particles, were recorded.

\subsection{High momentum protons and neutrons in asymmetric nuclei}

The events of type (e,e'p) and (e,e'n) were divided into two samples, corresponding to electron scattering off nucleons with high initial momentum $\left(p_{\text {miss }}>k_{F}\right)$ and low initial momen- 
tum $\left(p_{\text {miss }}<k_{F}\right)$. The nucleons, which had high momentum before the interaction, presumably belong to SRC pairs. And the low initial momentum nucleons likely form shell model states. The ratio between A(e,e'n) and A(e,e'p) events for both samples is shown in Fig. 4a. The ratio of knocked out neutrons to protons for low initial momenta grows as N/Z with nuclei mass number, as expected from simple nucleon counting. However, the (e,e'n)/(e,e'p) high momentum ratios are consistent with unity for all measured nuclei. Therefore, there are equal numbers of high-initial-momentum protons and neutrons in asymmetric nuclei, even though they contain up to $50 \%$ more neutrons than protons. This observation is consistent with the idea, that almost all high momentum nucleons within nucleus belong to SRC pairs, even in neutron rich nuclei. Another interesting conclusion is that in neutron rich nuclei the fraction of high-momentum protons is larger, than the fraction of high-momentum neutrons. Fig. $4 \mathrm{~b}$ shows the relative fraction of high momentum protons and neutrons in different nuclei. The double ratios of $\mathrm{A}\left(\mathrm{e}, \mathrm{e}^{\prime} \mathrm{N}\right)$ to carbon minimize the experimental and theoretical uncertainties for both $\mathrm{N}$ : protons and neutrons. The fraction of high momentum protons increases by about $50 \%$ from carbon to lead, whereas the corresponding fraction of high momentum neutrons decreases by about $10 \% \pm 5 \%$. The prediction of a simple phenomenological $n p$ dominance model, which uses a mean-field momentum distribution for low momenta nucleons $\left(p_{\text {miss }}<k_{F}\right)$ and a scaled deuteron-like tail for nucleons with high momenta $\left(p_{\text {miss }}>k_{F}\right)$, is shown in Fig. $4 \mathrm{~b}$ as well. This model agrees with the results. Further details on this analysis can be found in [5].
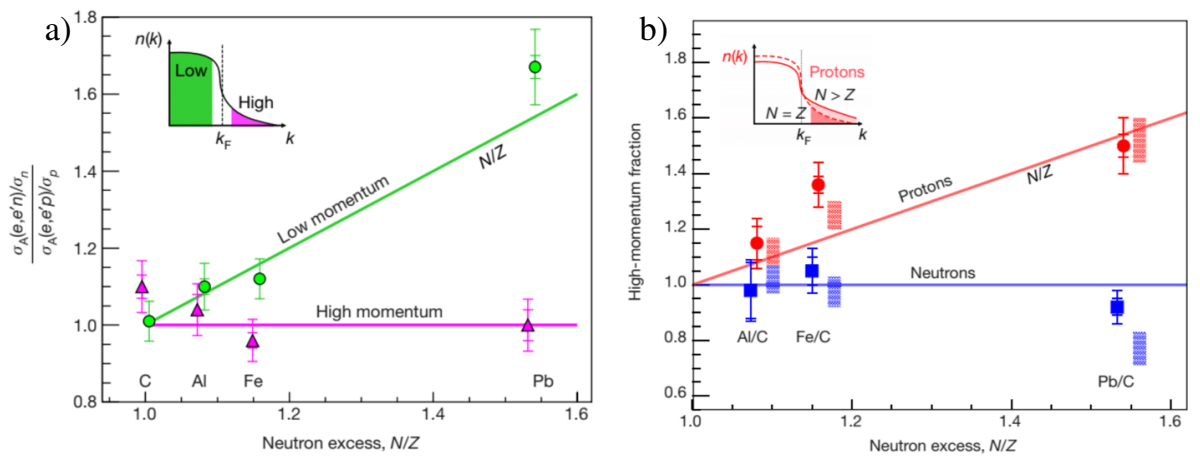

Figure 4. a) The ratios between neutrons and protons with high and low momenta for different nuclei are shown as $\left[\mathrm{A}\left(\mathrm{e}, \mathrm{e}^{\prime} \mathrm{n}\right) / \sigma_{n}\right] /\left[\mathrm{A}(\mathrm{e}, \mathrm{e}, \mathrm{p}) / \sigma_{p}\right]$ reduced cross-section ratios. Green circles and magenta triangles correspond to the samples with low and high initial momentum of the struck nucleon (see inset). b) Red circles show the double ratio of number of (e,e'p) events with proton high and low initial momentum for nucleus A relative to carbon. Red bands show the prediction of the phenomenological $n p$-dominance model. Blue squares and bands show the same for neutron knock out events. The inset demonstrates how adding neutrons increases the fraction of protons in the high momentum tail. For both plots the inner error bars are statistical, whereas the outer error bars include both statistical and systematic uncertainties. The lines show the simple N/Z expectation and a constant of 1 . Pictures are adapted from [5]

\subsection{The center-of-mass motion of SRC pairs}

The low c.m. momentum of an SRC pair and high momenta of both nucleons are fundamental characteristics, which indicate that the nucleons in the pair are in close proximity and limited interaction with the surrounding nuclear environment [8]. At high momenta, the 
momentum distribution of the SRC nucleons can be factorized into the c.m. and relative momentum distributions. This approach is used for effective calculations with scale separation, which agree with many-body calculations. Such scale separation implies, that the relative momentum distribution is a universal function of the short range part of the NN interaction. The nucleus-specific c.m. momentum distribution is then a direct probe for the nuclear manybody dynamics from which SRC pairs are formed.

The reaction of interest is A(e,e'pp), where events of type A(e,e'p) have a second, recoil proton with momentum $\left|\vec{p}_{\text {recoil }} \leq\right| 350 \mathrm{MeV} / c$. The recoil proton was emitted opposite to the leading one. The extracted width $\left(\sigma_{c . m}\right.$. $)$ of the center of mass momentum distribution shown in Fig. 5 is smaller than the Fermi momentum ranging from $140 \mathrm{MeV} / c$ to $170 \mathrm{MeV} / c$ for the measured nuclei. The extracted width is much smaller than the value of $\left|\vec{p}_{\text {miss }}\right|$ or $\left|\vec{p}_{\text {recoil }}\right|$, indicating that the nucleons within the $p p$-SRC pairs have large and opposite momentum. Further details on this analysis can be found in [6].

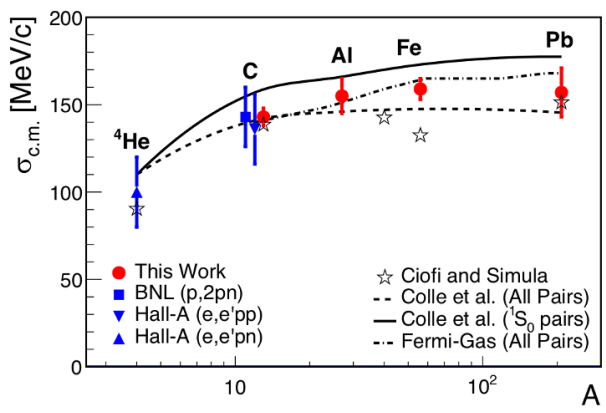

Figure 5. The width of the c.m. momentum distribution for SRC pairs as a function of nuclear mass. The red full circles show data points obtained in this work. These data agree with the previous measurements [12-14] (blue full squares and triangles). Theoretical calculations by Ciofi degli Atti and Simula [16] are shown as open stars, Colle et al. [15] considering all nucleon pairs as a dashed line, and only ${ }^{1} S_{0}$ pairs as a solid line. Calculations for SRC formation from the Fermi-gas is shown as a semi-dashed line. Figure is adapted from [6]

The measurement is in overall agreement with theoretical calculations [15, 16], which are based on the mean-field approach for the c.m. motion of the parent nucleons that create the SRC pairs. This supports the theoretical description, where SRC pairs are formed from temporal fluctuations of mean-field nucleons [17]. Theoretical models can form SRC pairs from nucleons in different states: all nucleon pairs are considered in [16] and [15], nucleons in relative ${ }^{1} S_{0}$ states form SRC in Ref. [15]. The data points lie somewhat inbetween of the two cases indicating some selectivity for nucleonic states, which can form SRC pairs.

\subsection{Modified structure of protons and neutrons in SRC pairs}

The European Muon Collaboration (EMC) effect [18] is now largely accepted as evidence that quark momentum distributions are different in bound nucleons relative to free nucleons [1921]. However, there is still no consensus as to the underlying nuclear dynamics driving it. Currently, there are two leading approaches for describing the EMC effect, which are both consistent with data: (A) all nucleons are slightly modified when bound in nucleus, (B) nucleons are unmodified most of the times, but are modified significantly when they fluctuate into SRC pairs [18]. The first experimental evidence supporting the second hypothesis is a 
linear correlation between the number of SRC pairs and the size of the EMC effect [22, 23]. Simultaneous measurement of the SRC cross sections and the EMC effect on deuterium and heavier nuclei were done using CLAS 6 data. This allowed determination of observables with better precision.

The EMC effect is characterized by the modification of the per-nucleon Deep Inelastic Scattering (DIS) cross-section or modification of the per-nucleon nuclear structure function, which is believed to be the same [20, 21]. The DIS data was selected based on $Q^{2}>1.5$ $\mathrm{GeV}^{2}$ and invariant mass of the proton plus virtual photon, $\mathrm{W}>1.8 \mathrm{GeV}$. The measured EMC effect, characterized as the approximately linear deviation from unity for $0.3 \leq x_{B} \leq$ 0.7, for different nuclei together with previous data is shown in Fig. 6a. This is the first measurement of EMC effect on lead.

We can model the modification of the nuclear structure function $F_{2}^{A}$ as entirely due to modification of $n p$-SRC pairs:

$$
\begin{aligned}
F_{2}^{A} & =n_{S R C}^{A}\left(F_{2}^{p *}+F_{2}^{n *}\right)+\left(Z-n_{S R C}\right) F_{2}^{p}+\left(N-n_{S R C}^{A}\right) F_{2}^{n} \\
& =Z F_{2}^{p}+N F_{2}^{n}+n_{S R C}^{A}\left(\Delta F_{2}^{p}+\Delta F_{2}^{n}\right),
\end{aligned}
$$

where $n_{S R C}^{A}$ is the number of SRC pairs in nucleus $A, F_{2}^{p}$ and $F_{2}^{n}$ are the free proton and neutron structure functions, $F_{2}^{p *}$ and $F_{2}^{n *}$ are the average modified structure functions for protons and neutrons in SRC pairs, and $\Delta F_{2}^{p}=F_{2}^{p *}-F_{2}^{p *}$ (and the same for $\Delta F_{2}^{p}$ ). $F_{2}^{p *}$ and $F_{2}^{n *}$ are assumed to be the same for all nuclei. We use the same parametrization for deuteron to extract $F_{2}^{n}$ and $F_{2}^{p} / F_{2}^{d}$ [26]. Fig. 6b shows the nucleus-independent part expressed in terms of nucleus-dependent variables from Eq. (1). Although the slope of EMC effect for different nuclei varies with $\mathrm{A}$, the ratio of SRC-modified structure functions is the same for all nuclei within uncertainties. Thus, we conclude, that the magnitude of the EMC effect in different nuclei can be described by the abundance of $n p$-SRC pairs and that the proposed SRC-pair modification is, in fact, universal. Further details on this analysis can be found in [7].

a)

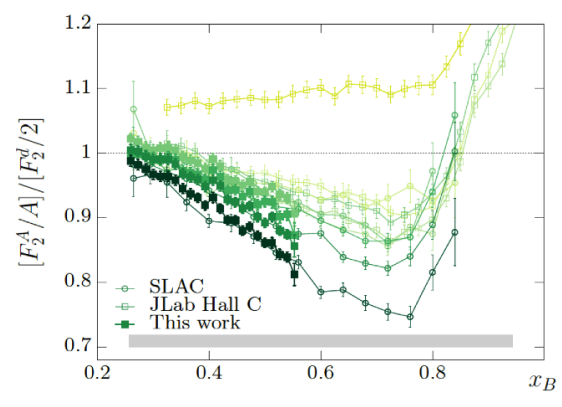

b)

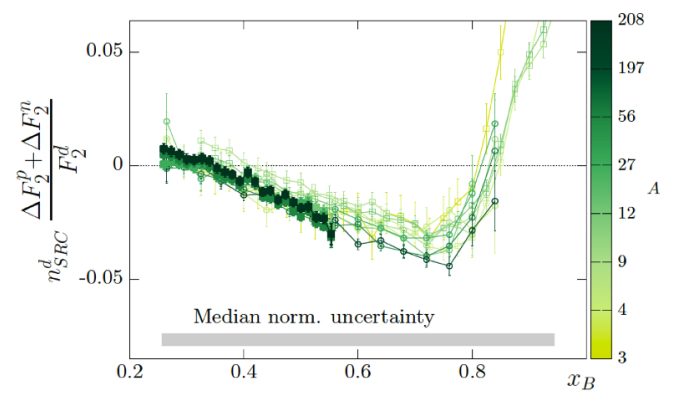

Figure 6. The EMC effect for different nuclei as ratios of structure functions $F_{2}^{A} / F_{2}^{d}$ (a) and the modification of SRC pairs (b) as a function of Bjorken variable $x_{B}$. Color marks the mass of the nucleus. The open circles are the SLAC data [24] and the open circles are the JLab data [25]. The gray bands show the median normalization uncertainty. Pictures are adapted from [7]

\section{Future experiments are designed for high precision measurements}

The work described in the previous section was done using only several hundreds of events on each target. The world SRC statistics currently accounts for several thousands of events. 
The new experiments are designed to add order of magnitude more data for better precision and sensitivity for various effects.

There is a number of upcoming SRC experiments planned at JLab, including a number of inclusive scattering measurements. These experiments aim on study how pair production change by adding protons or neutrons to the nucleus (electron scattering on ${ }^{40} \mathrm{Ca}$ and ${ }^{46} \mathrm{Ca}$, and ${ }^{5} 6 \mathrm{Fe}$ ), and studying the structure functions of protons and neutrons by tagging them with high momentum nucleon recoiling from a deuteron target. The hard exclusive scattering SRC program includes the CLAS-12 proposal, which will focus on simultaneous measurements of SRC and EMC effect and tag the recoil. It will use an $11 \mathrm{GeV}$ electron beam incident on a set of targets including liquid deuterium, helium, carbon, silicon, calcium, tin, and lead. The hard exclusive scattering on two isotopes of calcium: ${ }^{40} \mathrm{Ca}$ and ${ }^{46} \mathrm{Ca}$ will help to disentangle mass and asymmetry aspects of $n p$-dominance. The CLAS spectrometer will detect at the order of 10k events of both $n p$ and $p p$ pairs on each target.

The new SRC experiment at BM@N/Nuclotron setup (JINR) uses inverse kinematics, where a $4 \mathrm{GeV} / \mathrm{c}$ carbon beam hits the liquid hydrogen target. This makes it possible to study the properties of the nucleus after interaction. The BM@N spectrometer allows measuring four particles in the final state: both the scattered and the knocked out protons, the recoil and the residual nucleus. The data collected in March 2018 indicates, that we can identify different charge states of the residual nucleus, as shown in Fig 7. This measurement is expected to be the first in a series of SRC experiments at BM@N in inverse kinematics.

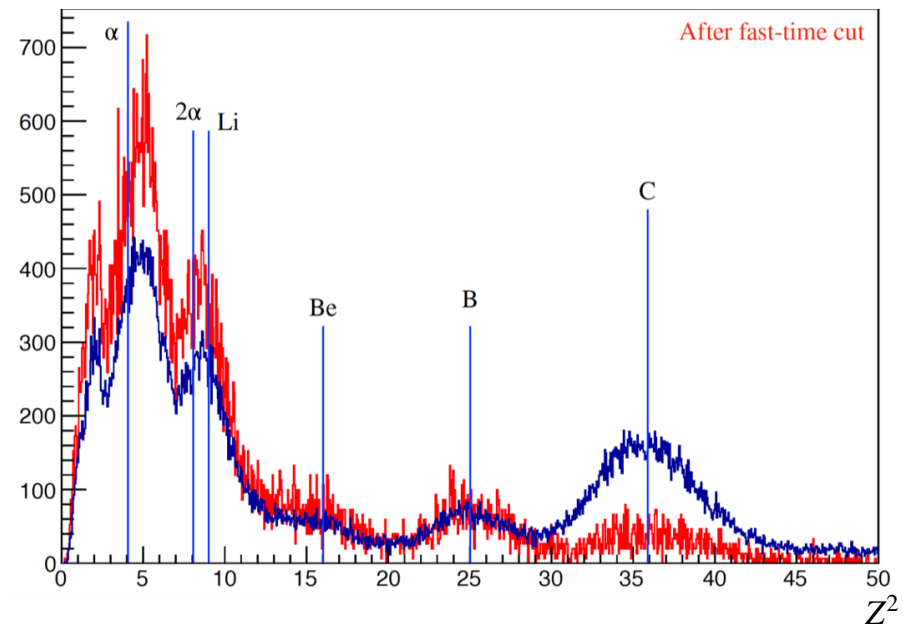

Figure 7. The squared charge of the residual nucleus. Vertical lines mark squared charge for particular nuclei. The blue spectrum shows all events, whereas the red line corresponds to the rough time cut for the leading and the scattered protons

\section{Conclusions}

Short Range Correlations is a vibrant field of studies on the border between nuclear and particle physics. Hard exclusive scattering reactions is one of the effective ways to study the SRC, where all particles produced in the interaction are detected. The recent analyses of the CLAS 6 data confirmed $n p$-dominance over a wide range of nuclei masses, provided a valuable insight on the SRC formation process, and strengthened the hypothesis that EMC effect is 
caused by strongly modified SRC nucleons. The upcoming experiments based on hard exclusive reactions are designed to perform high-statistics precise measurements of SRC aiming among other things at disentangling mass and asymmetry aspects of the $n p$-dominance and studying the properties of the residual nucleus after interaction.

\section{References}

[1] A. Tang et al., Phys. Rev. Lett. 90, 042301 (2003)

[2] E. Piasetzky et al., Phys. Rev. Lett. 97, 162504 (2006)

[3] Shneor et al.,Phys. Rev. Lett. 99, 072501 (2007)

[4] Subedi et al., Science 320, (2008)

[5] M. Duer et al., Nature 560, 617 (2018)

[6] E. O. Cohen et al., Phys. Rev. Lett. 121, 092501 (2018)

[7] B. Schmookler, submitted for publication

[8] R. Weiss, et al., Phys. Lett. B 780, 211 (2018)

[9] R. B. Wiringa et al., Phys. Rev. C 89, 024305 (2014)

[10] J. Carlson et al., Rev. Mod. Phys. 87, 1067 (2015)

[11] T. Neff et al., Phys. Ref. C 92, 024003 (2015)

[12] A. Tang et al., Phys. Rev. Lett. 90, 042301 (2003)

[13] R. Schneor et al., Phys. Rev. Lett. 99, 072501 (2007)

[14] I. Korover et al., Phys. Rev. Lett. 113, 022501 (2014)

[15] C. Colle et al., Phys. Rev. C 89, 024603 (2014)

[16] C. Ciofi degli Atti and S. Simula, Phys. Rev. C 53, 1689 (1996)

[17] O. Hen et al., Rev. Mod. Phys, 89, 045002 (2017)

[18] J. Aubert et al., Phys. Lett. B 123, 275 (1983)

[19] O. Hen et al., Rev. Mod. Phys. 89, 045002 (2017)

[20] P. R. Norton, Rep. Prog. Phys. 66, 1253 (2003)

[21] S. Malace et al., Int. J. Mod. Phys. E 23, 1430013 (2014). [arXiv:1405.1270]

[22] L. Weinstein et al., Phys. Rev. Lett. 106, 052301 (2011)

[23] O. Hen et al., Phys. Rev. C 85, 047301 (2012)

[24] J. Gomez et al., Phys. Rev. D 49, 4348 (1994)

[25] J. Seely et al., Phys. Rev. Lett. 103, 202301 (2009)

[26] J. Arrington et al., J. Phys. G 36, 025005 (2009). [arXiv:0805.3116] 